\title{
ONE MONTH-TADALAFIL 5 MG ONCE DAILY ADMINISTRATION \\ IMPROVES CLINICAL AND ULTRASOUND PARAMETERS \\ OF CHRONIC PELVIC PAIN SYNDROME/LOWER URINARY TRACT SYMPTOMS
}

\section{Lotti F.', Corona G. ${ }^{1}$, Maggi M. ${ }^{1}$}

${ }^{1}$ Sexual Medicine and Andrology Unit, Department of Experimental and Clinical Biomedical Sciences, University of Florence, Florence, Italy;

Introduction.

-Tadalafil $5 \mathrm{mg}$ once daily (T5od) has been recently approved by the Food and Drug Administration for the treatment of lower urinary tract symptoms (LUTS), along with erectile dysfunction (ED).

-Recent pooled data analyses demonstrated that T5od-IPSS related improvements were significant regardless of confounders.

-PDE5 inhibitors exert a positive effect on ejaculatory latency.

We assessed the clinical outcome of 30 days-T5od administration on a patient complaining of CPPS/LUTS.

Case report. A Caucasian men of 27 years presented for chronic pelvic pain syndrome (CPPS) and LUTS.

He has been complaining since one month of CPPS, LUTS, mild ED and acquired premature ejaculation.

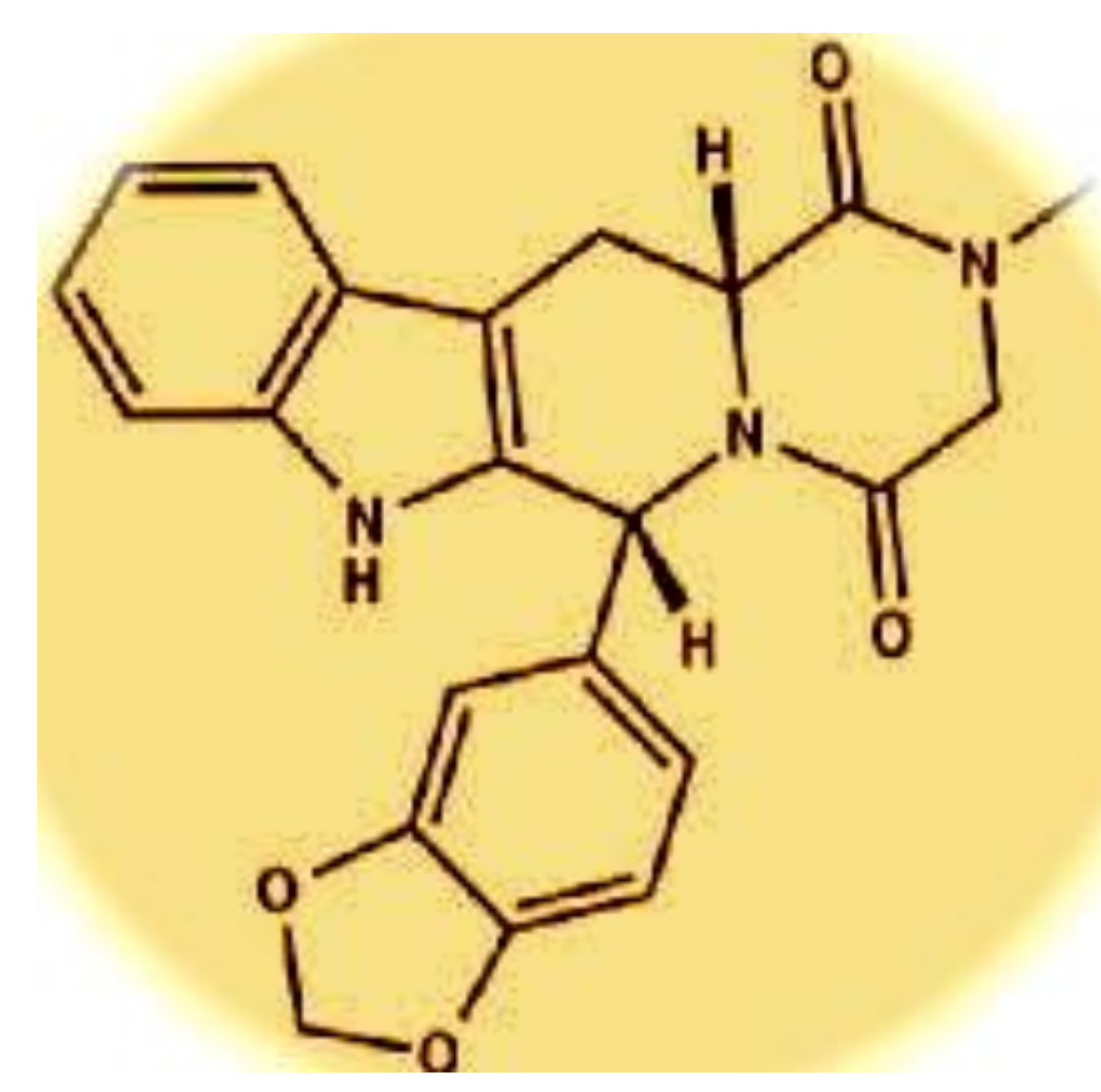

Tadalafil He underwent, before and after treatment:

-medical history assessment,

-complete physical examination,

-scrotal and transrectal color-Doppler ultrasound (CDUS),

before and after ejaculation,

-semen analysis including seminal interleukin 8 (sIL-8) measurement, -validated questionnaires exploring:

1.CPPS (NIH-Chronic Prostatitis Symptom Index, NIH-CPSI),

2.LUTS (International Prostate Symptom Score, IPSS),

3.sexual and erectile function (IIEF-15; IIEF-15-erectile function domain), 4.ejaculatory status (Premature Ejaculation Diagnostic Tool, PEDT),

5.psychological symptoms (Middlesex Hospital Questionnaire).

\begin{tabular}{|l|c|}
\hline \multicolumn{2}{|c|}{ Clinical parameters } \\
\hline Age & 27 \\
\hline Body mass index $\left(\mathrm{kg} / \mathrm{m}^{2}\right)$ & 24.2 \\
\hline Systolic/diastolic blood pressure $(\mathrm{mmHg})$ & $120 / 80$ \\
\hline Smoking habit & Current smoker \\
& $(5$ cigarettes/day) \\
\hline Alcohol assumption & Occasional \\
\hline Physical activity & No \\
\hline Total testosterone (nmol/L) & 27.6 \\
\hline Current positive seminal and/or urine cultures & No bacteria \\
\hline Mean testis volume (Prader) (ml) & 23.5 \\
\hline Epididymal abnomalities & left head: palpable nodule/cyst \\
\hline Prostate pain on palpation & yes \\
\hline
\end{tabular}

After treatment the patient showed an improvement in NIH-CPSI and IPSS scores.

Global sexual and erectile functions, assessed by IIEF-15 and IIEF-15-EFD, respectively, improved,

as well as ejaculatory latency (see PEDT score).

Psychological status, assessed by MHQ, showed a consistent amelioration at the end of the treatment.

\begin{tabular}{|l|c|c|}
\hline \multicolumn{1}{|c|}{ Questionnaires scores } & $\begin{array}{c}\text { Pre- } \\
\text { Tadalafil }\end{array}$ & $\begin{array}{c}\text { Post- } \\
\text { Tadalafil }\end{array}$ \\
\hline Questionnaires score & & $\mathbf{1 7}$ \\
\hline NIH-CPSI (0-43) & $\mathbf{2 2}$ & $\mathbf{1 2}$ \\
\hline -pain subdomain (0-21) & $\mathbf{5}$ & $\mathbf{3}$ \\
\hline -void subdomain (0-10) & $\mathbf{5}$ & $\mathbf{4}$ \\
\hline -quality of life subdomain (0-12) & $\mathbf{2 3}$ & $\mathbf{1 4}$ \\
\hline IPSS (0-40) & $\mathbf{5 8}$ & $\mathbf{6 8}$ \\
\hline IIEF-15(5-75) & $\mathbf{2 4}$ & $\mathbf{2 7}$ \\
\hline IIEF-15-erectile function domain (1-30) & $\mathbf{1 4}$ & $\mathbf{1 1}$ \\
\hline PEDT (0-20) & $\mathbf{1 9}$ & $\mathbf{7}$ \\
\hline MHQ (0-96) & & \\
\hline
\end{tabular}

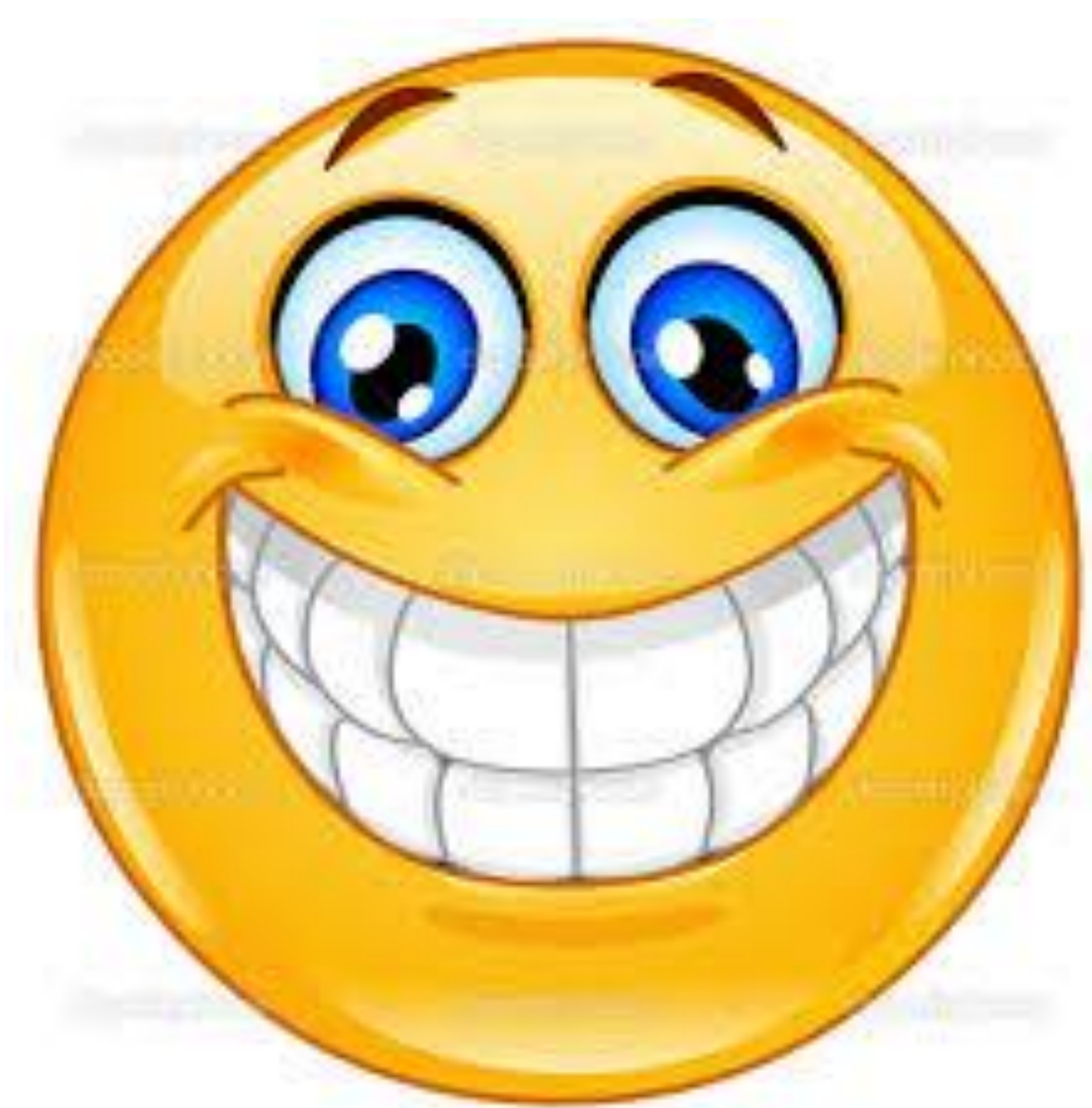

We also observed an improvement in biochemical and CDUS parameters related to prostate-vesicular inflammation. In particular, a reduction in sIL-8 levels and a significant improvement in arterial peak systolic velocity, parameters related to prostate inflammation and CPPS, were observed after treatment.

\begin{tabular}{|l|c|c|}
\hline $\begin{array}{c}\text { Color-Doppler ultrasound parameters } \\
\text { and sIL-8 levels }\end{array}$ & $\begin{array}{c}\text { Pre- } \\
\text { Tadalafil }\end{array}$ & $\begin{array}{c}\text { Post- } \\
\text { Tadalafil }\end{array}$ \\
\hline Prostate volume (ml) & $\mathbf{3 0}$ & $\mathbf{2 7}$ \\
\hline Prostate echo-texture abnormalities & no & no \\
\hline Prostate arterial peak systolic velocity (cm/s) & $\mathbf{1 2 . 7}$ & $\mathbf{6 . 8}$ \\
\hline Prostate hyperaemia & yes & no \\
\hline Seminal vesicles volume before ejaculation (ml) & $\mathbf{3 6 . 7 8}$ & $\mathbf{3 0 . 8 6}$ \\
\hline Seminal vesicles volume after ejaculation (ml) & $\mathbf{3 3 . 1 3}$ & $\mathbf{2 7 . 8 3}$ \\
\hline Seminal vesicles ejection fraction (\%) & 9.9 & 9.8 \\
\hline Seminal vesicles echo-texture abnomalities & yes & yes \\
\hline Testis mean volume (ml) & 16 & 16 \\
\hline Epididymal head (mm) & 9.75 & 9.5 \\
\hline Epididymal tail (mm) & 5.5 & 5.4 \\
\hline Epididymal echo-texture abnormalities & no & no \\
\hline Epididymal hyperaemia & no & no \\
\hline Varicocele & no & no \\
\hline Seminal interleukin 8 (ng/ml) & $\mathbf{8 . 1 5}$ & $\mathbf{6 . 1 4}$ \\
\hline
\end{tabular}
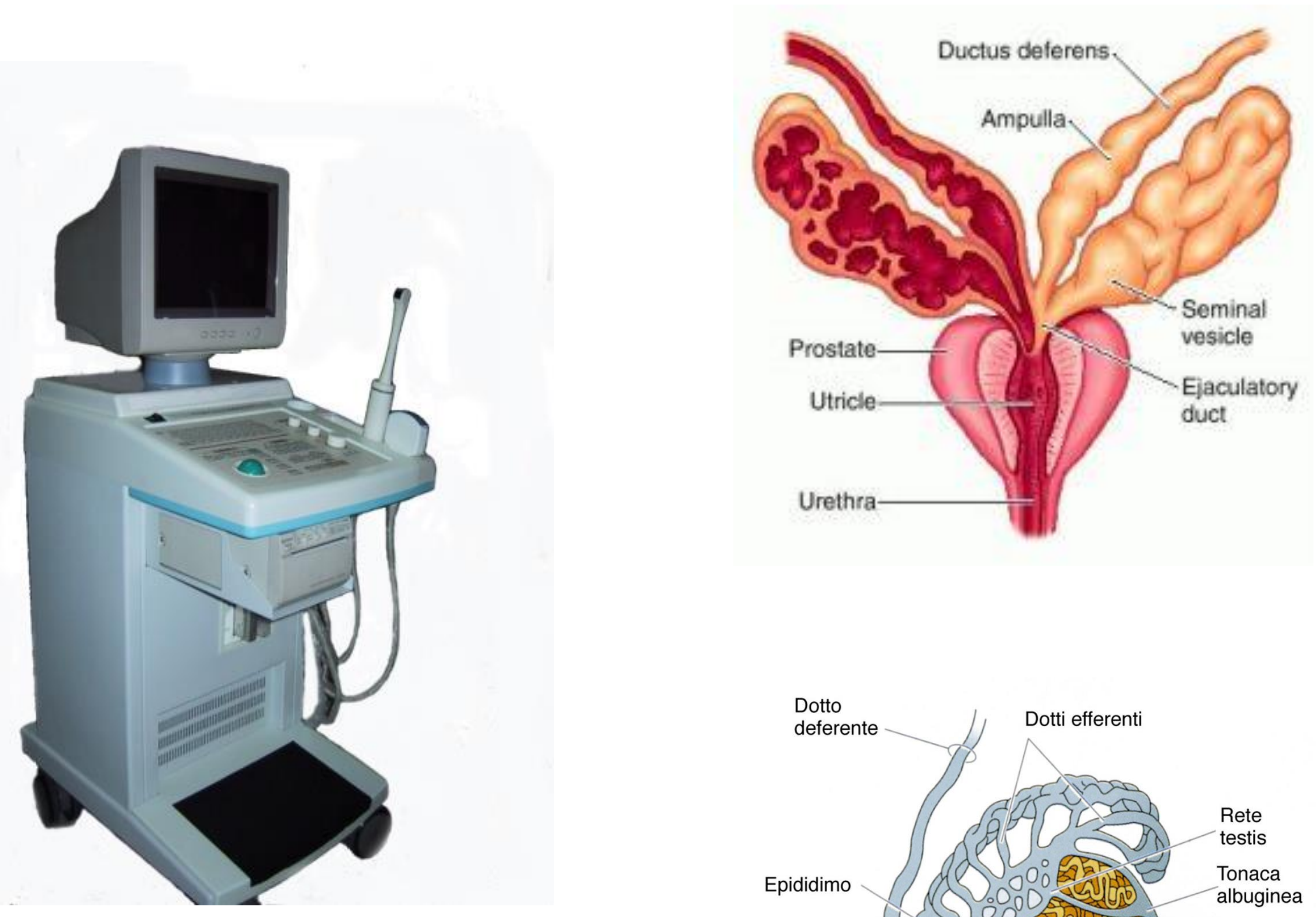

Conclusion. A 30 days-T5od administration seems to exert an improvement in CPPS/LUTS, biochemical and CDUS parameters of prostate inflammation, along with psycho-sexual function. 